\title{
TARMED-Volumen pro Patient seit Jahren stabil
}

\author{
Kerstin Schutz, Sabine Zehnder \\ Expertinnen Abteilung Ambulante Versorgung und Tarife
}

1 Diese Berechnung bezieht sich auf alle Fachrichtungen, die Gesetze KVG, UVG, IVG, MVG und alle Tarife für diese Gesetze.

2 Die Corona-Pandemie sorgt für Mehrkosten von ger/Prantl. Schweiz Ärzteztg. 2020;101(44):1442-4

3 Unter der Gruppe «Invasiv» werden folgende Fachrichtungen zusammengefasst Chirurgie, Gynäkologie und Geburtshilfe, Handchirurgie, Ophthalmologie, OtoRhino-Laryngologie, Orthopädie, Plastische Chirurgie, Urologie.

4 Unter der Gruppe «Grundversorger» werden folgende Fachrichtungen zusammengefasst: Allgemeine Innere Medizin, Kinderund Jugendmedizin, Prak tische Ärzte.

5 Unter der Gruppe «Spezialärzte» werden folgende Fachrichtungen zusammengefasst: Angiologie, Dermatologie, Endokrinologie, Gastroenterologie, Hämatologie, Infektiologie, Kardiologie, Medizinische Onkologie, Nephrologie, Neurologie, Physikalische Medizin, Pneumologie, Rheumatologie. CHF 735 Millionen. Oesch-

\section{Die Abrechnungsdaten 2020 der ärzteeigenen Datensammlung liegen vollständig} vor. Betrachtet man das TARMED-Volumen pro Praxis, endet das Jahr mit einem Verlust im Vergleich zu 2019. Die Effekte des Behandlungsverbotes und des Lockdowns konnten je nach Fachdisziplin bis Ende des Jahres mit deutlicher Mehrarbeit teilweise kompensiert werden.

Die Aussichten im April 2020 sahen düster aus: Im praxisambulanten Bereich verbuchten Praxen je nach Disziplin bis zu 40\% Verlust des TARMED-Abrechnungsvolumens im Vergleich zum Vorjahr, die Patientenkontakte gingen um einen ähnlichen Anteil zurück (Abb. 1). Insgesamt lag der Umsatzrückgang ${ }^{1}$ pro Praxis im zweiten Quartal durchschnittlich bei circa $14 \%$. Weiterhin stellten die Mehrkosten ${ }^{2}$, die durch die erhöhten Schutzmassnahmen sinnvollerweise getroffen werden mussten, die Ärzteschaft vor grosse Herausforderungen.

Die Fachdisziplinen waren von den Massnahmen unterschiedlich betroffen: Die invasiv tätige Ärzteschaft ${ }^{3}$ beispielsweise konnte in der Zeit des Lockdowns knapp die Hälfte der Behandlungen nicht durchführen. Die Eingriffe konnten jedoch mit erheblicher Mehrarbeit zum Teil nachgeholt werden, und so konnte der Verlust vom Frühling etwas aufgefangen werden. Für den invasiven Bereich liegt der durchschnittliche TARMEDVerlust für das Jahr 2020 insgesamt bei 3,12\%.

\section{Kennzahlen 2020 im Vergleich zum Vorjahr}

$$
-1.87 \%
$$

TARMED Volumen pro Sitzung

\section{$-1.41 \%$} Patienten pro Praxis

\section{Dürres Frühjahr, starker Juni}

Auch in der Grundversorgung ${ }^{4}$ und bei den Spezialärztinnen und -ärzten ${ }^{5}$ fragten die Patienten, die während der Behandlungsrestriktionen nicht dringlich behandelt werden mussten, nach dem Lockdown die ärztlichen Leistungen wieder nach. So liegt beispielsweise im Juni 2020 das TARMED-Volumen pro Praxis 20\% über dem Vorjahreswert (Abb. 2).

Die Nachfrage nach psychiatrischen Leistungen hat hingegen 2020 insgesamt zugenommen. Diese Zunahme gilt für neue und für bestehende Patienten, wobei die Erstkontakte sogar während des Lockdowns im März und April 2020 leicht zugenommen haben. Dies erklärt das leicht erhöhte TARMED-Volumen im Vergleich zum Vorjahr (Abb. 2).

Insgesamt schliesst das Jahr 2020 über alle Facharztdisziplinen mit einem durchschnittlichen Verlust im Bereich TARMED von 1,15\% pro Praxis (Abb. 1). Die Höhe des Verlustes differiert je nach Disziplin: Er liegt beispielsweise in der Kinder- und Jugendmedizin, ORL, Pneumologie und Nephrologie bei über $8 \%$. Hingegen verzeichnen Rheumatologie, Psychiatrie und Radiologie einen Zuwachs des TARMED-Volumens pro Praxis von circa 3 bis $4 \%$.

\section{Weniger Erstkontakte}

Das durchschnittliche TARMED-Volumen pro Sitzung sinkt um knapp 2\% (Abb. 1), was sich auf die Verlagerung zu telemedizinischen Leistungen vor allem während der Monate März bis Mai zurückführen lässt. Entgegen der üblichen leichten Zunahme der Anzahl Erstkontakte pro Praxis (Patienten pro Praxis) pro Jahr, nahm selbst diese Kennzahl im Jahr 2020 um gut 1,4\% ab (Abb. 1). 


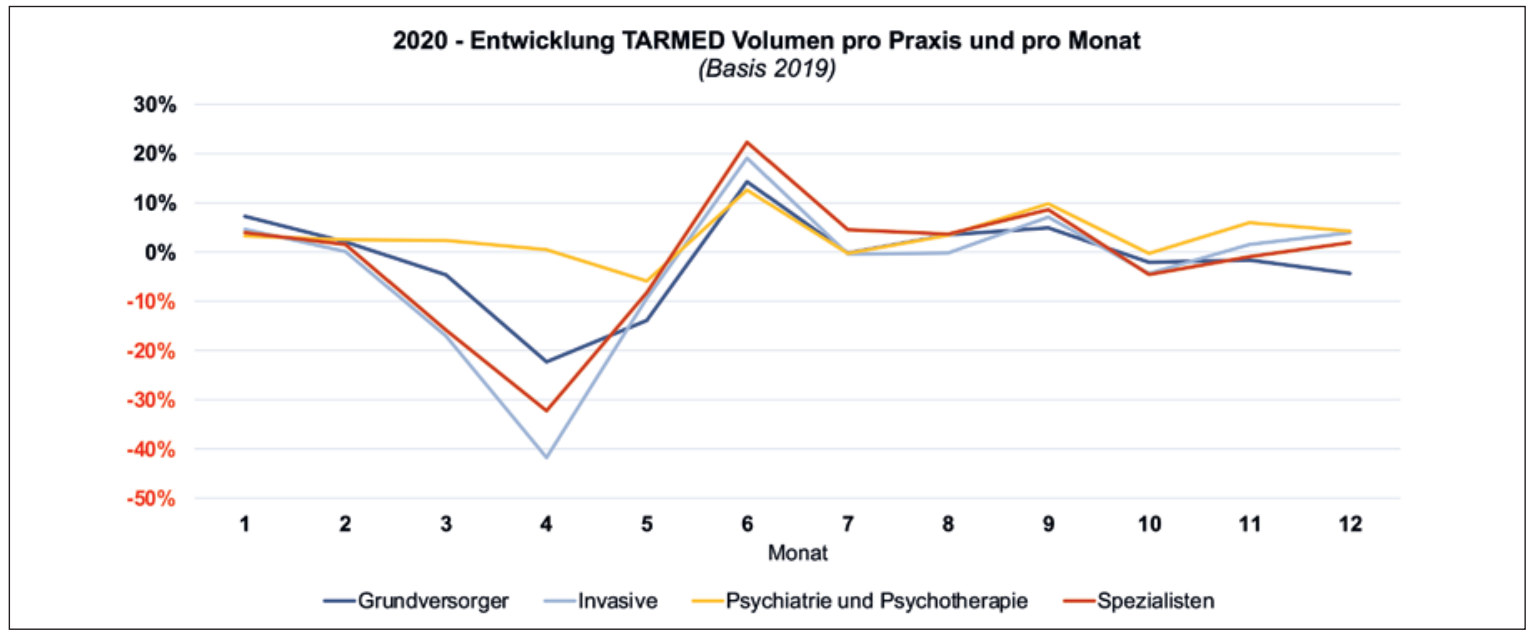

Abbildung 2: TARMED-Volumen (Taxpunkte) 2020 pro Praxis/pro Monat nach Fachgruppe im Vergleich zum Vorjahr, Gesetz: KVG, Geographie: $\mathrm{CH}$, Hochrechnung: Nein.

Das durchschnittliche TARMED-Volumen pro Patientin und Patient bleibt seit 2017 stabil (Abb. 3). Dies bedeutet, dass die behandelten Patienten auch unabhängig von der Pandemiesituation ärztliche Leistungen benötigen.

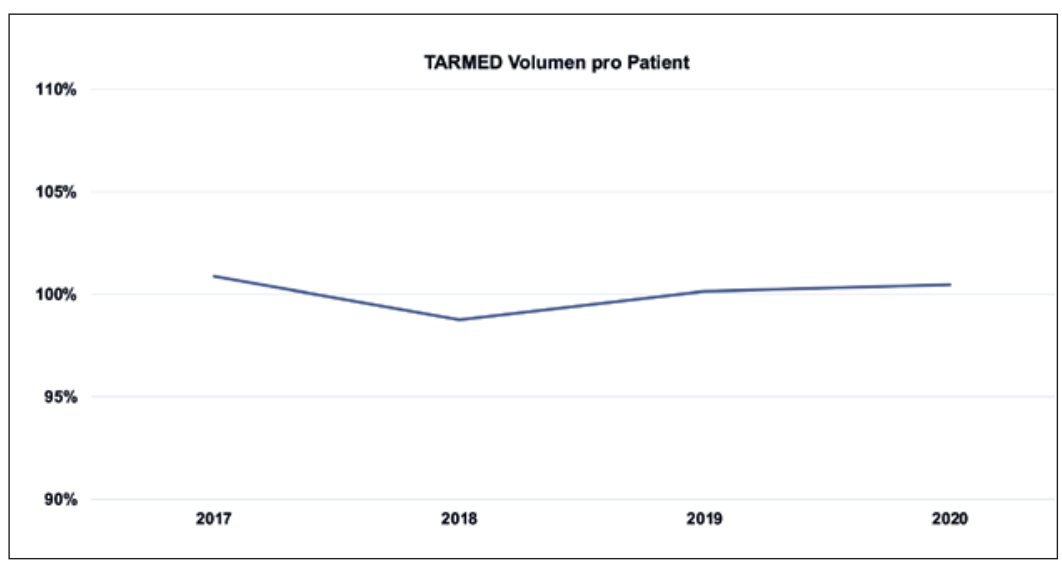

Abbildung 3: TARMED-Volumen (Taxpunkte) pro Patient, pro Jahr, alle Facharztdisziplinen, Gesetz: KVG, Geographie: $\mathrm{CH}$, Hochrechnung: Nein.

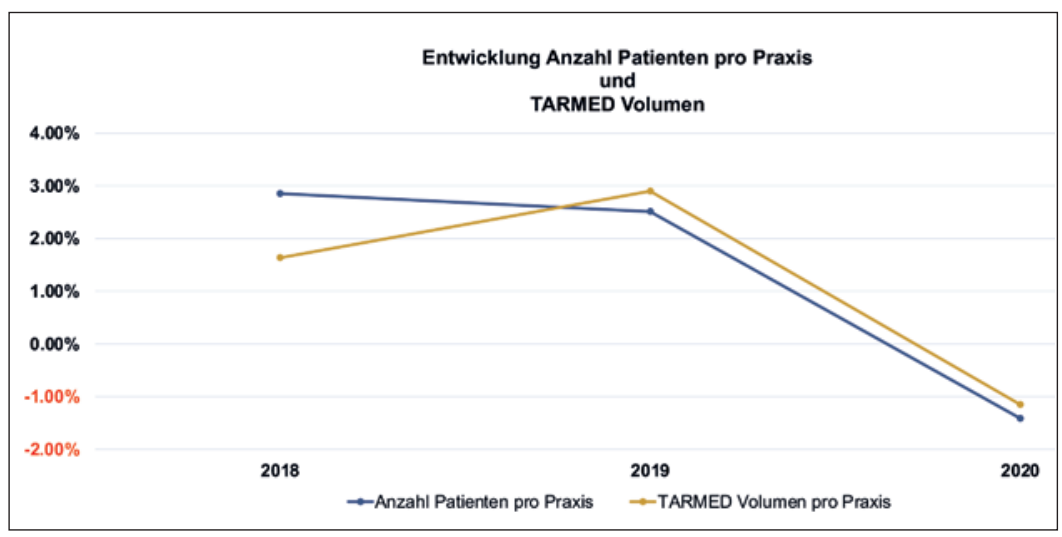

Abbildung 4: Entwicklung TARMED-Volumen (Taxpunkte) und Anzahl Patienten pro Praxis, pro Jahr, alle Facharztdisziplinen, Gesetz: KVG, Geographie: $\mathrm{CH}$, Hochrechnung: Nein.
Dies bedeutet auch, dass keine Kompensation des Tarifeingriffs von 2018 durch Mengenausweitung erfolgte. Die Zunahme des TARMED-Gesamtvolumens ist auf die Zunahme der Erstkontakte zurückzuführen. Abbildung 4 verdeutlicht diesen Effekt aus anderer Perspektive: Das TARMED-Volumen pro Praxis entwickelt sich kongruent mit der Anzahl Patienten pro Praxis. Eine Kostensteigerung oder Kostensenkung hängt direkt mit der Anzahl Patienten zusammen, deren Nachfrage nur sehr eingeschränkt durch die Ärzteschaft steuerbar ist.

\section{Mehr Telemedizin}

Die Verlagerung von physischen Konsultationen zu telemedizinischen Konsultationen konnte im gesamten Jahr 2020 beobachtet werden. Diese Entwicklung ist über alle Fachrichtungen mit unterschiedlicher Ausprägung sichtbar. Während im Bereich Psychiatrie eine zum Teil hohe Verlagerung stattfindet, ist dies für die Spezialärztinnen und -ärzte nur für bestimmte Leistungen möglich.

Abbildung 5 zeigt deutlich, dass in den Monaten März bis Mai 2020 die telemedizinischen Konsultationen nicht zusätzlich zu physischen Konsultationen nachgefragt wurden, vielmehr haben diese die nicht mehr möglichen physischen Konsultationen teilweise kompensiert.

Der Aufwand für Leistungen in Abwesenheit des Patienten nimmt in den letzten Jahren stetig zu, so verständlicherweise auch im Pandemiejahr 2020. Die abgerechneten Leistungen in Abwesenheit steigen aber in einem moderaten Mass von 6,16 Taxpunkten (TP) auf 6,87 (TP) in einer durchschnittlichen Sitzung. Bei einer Höhe von 108,67 TP für eine durchschnittliche 


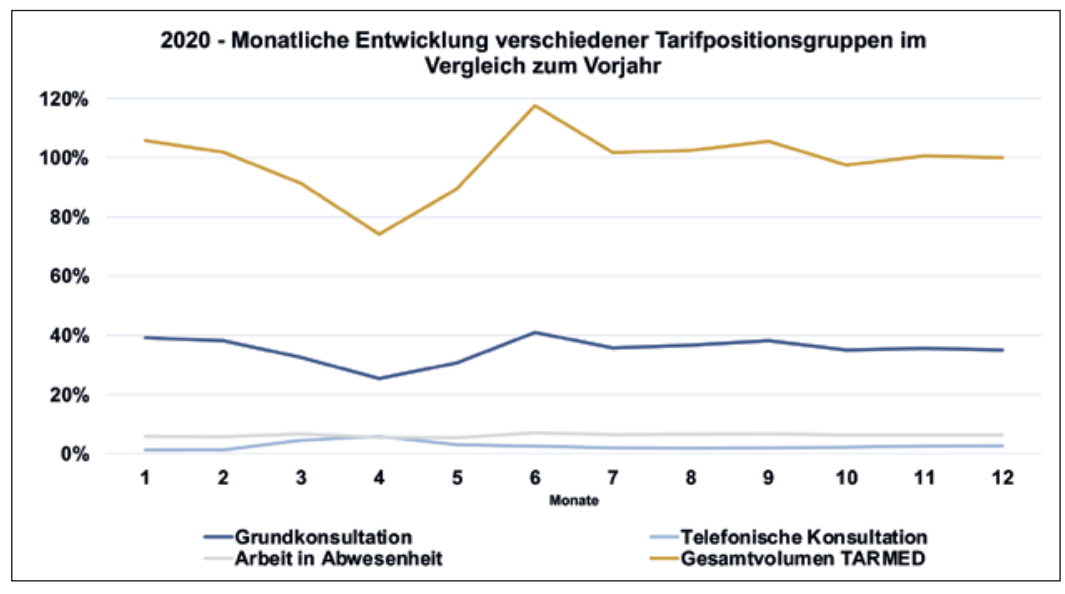

Abbildung 5: Entwicklung von Tarifpositionsgruppen (Volumen in Taxpunkten) 2020, pro Monat, alle Facharztdisziplinen, Gesetz: KVG, Geographie: $\mathrm{CH}$, Hochrechnung: Nein.

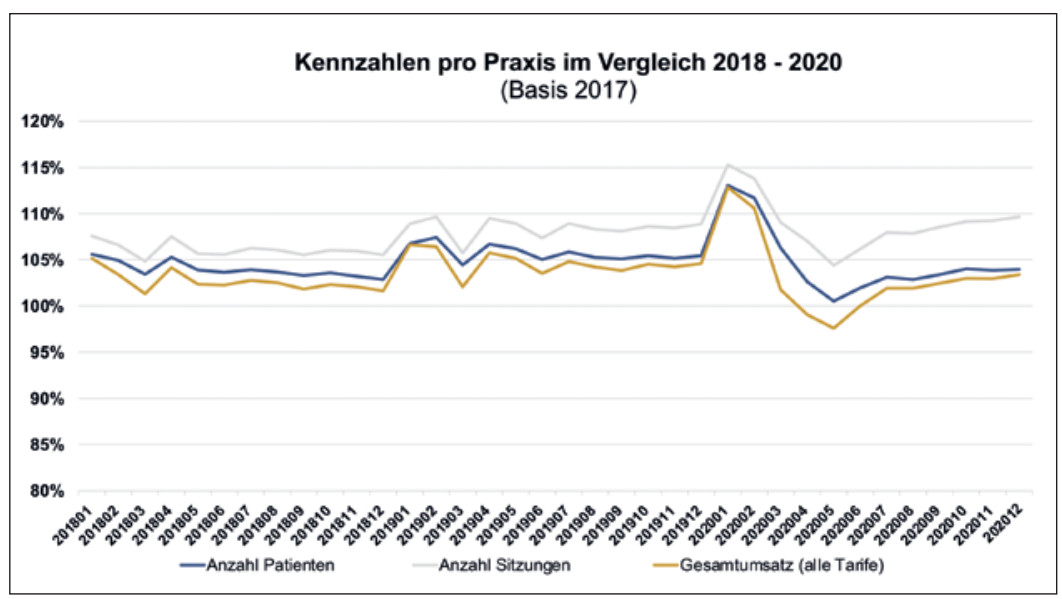

Abbildung 6: Kennzahlen im Vergleich 2018-2020, Basisjahr 2017, alle Facharztdisziplinen, Gesetz: KVG, Geographie: $\mathrm{CH}$, Hochrechnung: Nein.

\section{Die Datenlieferung ent- spricht dem NAKO-Kodex. Der NAKO-Kodex regelt den Zugriff sowie die Nutzung der Daten durch Verbände und Dritte. Dabei werden aggregierte Umsatzdaten einer Durchschnittspraxis zur Verfügung gestellt.}

FMH/Abteilung Ambulante Versorgung und Tarife Baslerstrasse 47 CH-4600 Olten Tel. 0313591230 Fax 0313591238 tarife.ambulant[at]fmh.ch

\section{Viele sistierte Behandlungen nachgeholt}

Die Analyse des TARMED-Volumens insgesamt sowie weiterer dazugehöriger Kennzahlen (Abb. 6) wie beispielsweise der Anzahl Patienten spiegelt die Pandemiesituation deutlich wider:

Nach der starken Reduktion in den Frühlingsmonaten (der Umsatz pro Praxis sinkt deutlich unter den Wert von 2017) konnten viele Praxen durch deutlichen Mehraufwand Behandlungen nachholen. Jedoch ist für nahezu alle Fachrichtungen ein Rückgang im Gesamtvolumen zu beobachten und das Vorjahresniveau wird nicht erreicht.

Das TARMED-Volumen pro Patientin und pro Patient ist analog der Vorjahresentwicklungen im Jahr 2020 konstant, was bedeutet, dass in den Sommer- und Herbstmonaten nur Behandlungen und Eingriffe nachgeholt wurden, die im Frühjahr nicht durchgeführt werden konnten.

\section{Solide Analysen dank der ärzteeigenen Datensammlung}

Dank der ärzteeigenen Datensammlungen können umfassende Analysen von Abrechnungsdaten im praxisambulanten Bereich vorgenommen werden. In den letzten Jahren haben circa 10000 Arztpraxen pro Jahr aktiv Daten geliefert und durch Beiträge an die kantonalen Trustcenter die Datensammlung finanziert. Nachdem die Société Vaudoise de Médecine (SVM) im Kanton Waadt und Teile der Innerschweiz eigene Lösungen verfolgen, rechnen wir im praxisambulanten Bereich mit einer schweizweiten $A b-$ deckung von rund 65\%. Im Datenpool der Schweizer Ärzteschaft sind aktuell 316 Mio. Rechnungskopien mit rund 3,2 Milliarden Leistungsdatensätzen (Stand: Ende März 2021) vorhanden. Die vorhandenen Daten sind grundsätzlich in jeder auf einer Rechnung verfügbaren Dimension auswertbar. Weiterhin kann analysiert werden, welche Leistungen häufig gemeinsam in einer Sitzung erbracht werden oder aus welchen Tarifpositionen sich eine durchschnittliche Sitzung zusammensetzt.

Die umfassenden Analysen sind nur dank jener Arztpraxen möglich, welche ihre Abrechnungsdaten an die kantonalen Trustcenter liefern. Die Zahl der Datenlieferanten nimmt stetig zu, und es ist unser Ziel, die Abdeckung weiter zu erhöhen. Wir bedanken uns bei allen Ärztinnen und Ärzten, die mit ihrer Datenlieferung die Datensammlung verbessert haben, sie mitfinanzieren und es der FMH damit ermöglichen, so aussagekräftige Analysen durchzuführen.

Seit Beginn des Jahres 2021 arbeitet das Schweizer Staatssekretariat für Wirtschaft (SECO) mit der FMH zusammen: Die FMH liefert künftig auf die gesamte Schweiz aggregierte Umsatzdaten an das SECO ${ }^{6}$. Die Daten dienen dem SECO zum Zweck der Konjunkturbeobachtung im Gesundheitswesen. Wir freuen uns sehr über diese Zusammenarbeit, welche die Bedeutung der ärzteeigenen Datensammlung unterstreicht. 